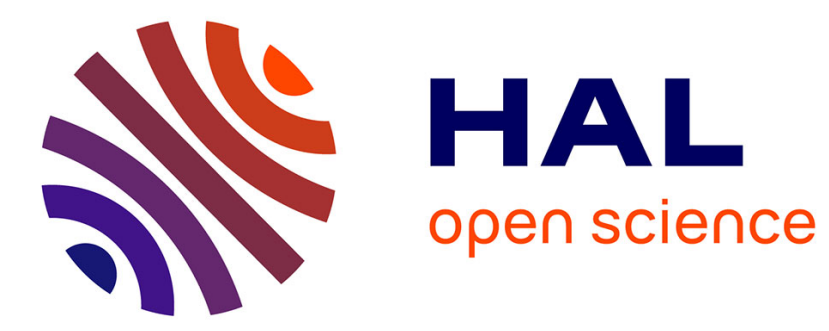

\title{
Structures productives de l'industrie du transport urbain et effets des schémas réglementaires \\ Philippe Gagnepain
}

\section{To cite this version:}

Philippe Gagnepain. Structures productives de l'industrie du transport urbain et effets des schémas réglementaires. Economie et Prévision, 1998, 135, pp.95-107. hal-00622819

\section{HAL Id: hal-00622819 https://hal.science/hal-00622819}

Submitted on 13 Sep 2011

HAL is a multi-disciplinary open access archive for the deposit and dissemination of scientific research documents, whether they are published or not. The documents may come from teaching and research institutions in France or abroad, or from public or private research centers.
L'archive ouverte pluridisciplinaire HAL, est destinée au dépôt et à la diffusion de documents scientifiques de niveau recherche, publiés ou non, émanant des établissements d'enseignement et de recherche français ou étrangers, des laboratoires publics ou privés. 


\title{
Structures productives de l'industrie du transport urbain et effets des schémas réglementaires ${ }^{1}$
}

\author{
Philippe Gagnepain \\ GREMAQ, Université des Sciences Sociales, Toulouse. \\ Première Version: Avril 1996 \\ Cette Version: Septembre 1998
}

Résumé

Increasing attention is being paid to the economic underpinnings of public transit services. Issues pertaining to the transport operator's production and cost structures, as well as the effects of regulation and subsidy policies on the operator's performance are attracting growing interest. The objective of this paper is to estimate a Translog cost function using observations on 60 different urban areas and contractual relationships between local authorities and public or private operators between 1985 and 1993. The results suggest that local authorities usually set prices above marginal costs, but below average costs, in order to respond to universal service obligations. Second, it is shown that operators' costs are lower when they are regulated under incentive contracts (as opposed to cost plus contracts), i.e., under regimes that give operators perfect incentives to provide more effort and reduce operating costs.

Une attention croissante est accordée aux enjeux économiques des services de transport en commun. Les questions relatives à la production et les coûts de transport, ainsi qu'aux effets de la réglementation et des politiques de subventions sur les performances de l'opérateur sont d'un intérêt croissant. L'objectif de cet article est d'estimer une fonction de coût translog à partir d'observations sur les relations contractuelles entre les autorités locales et les opérateurs publics ou privés dans 60 réseaux urbains entre 1985 et 1993. Les résultats suggèrent que les autorités locales fixent en général des prix supérieurs aux coûts marginaux, mais inférieurs aux coûts moyens, afin de répondre aux obligations de service universel. Ensuite, il est suggéré que les coûts des opérateurs sont inférieurs lorsque la réglementation est assurée par des contrats incitatifs (par opposition aux contrats de type coût du service), c'est à dire, par des régimes qui donnent aux opérateurs des incitations parfaites en termes d'effort à la réduction des coûts d'exploitation. 


\section{Introduction}

Le transport urbain français est une industrie fortement réglementée. Chaque réseau de transport est géré par une autorité locale qui a la responsabilité de l'organisation du transport sur son territoire. Le service est assuré par un opérateur généralement privé ou semi-public. Le contenu du contrat qui lie l'autorité organisatrice (A.O.) et l'exploitant définit, à travers la réglementation mise en place, la nature du financement de l'exploitation et donc les risques industriels (risques liés aux coûts) et commerciaux (risques liés aux recettes) encourus par l'opérateur. On dénombre environ six types de contrats en France dont trois sont largement majoritaires ${ }^{2}$ : Les contrats de type gérance, gestion à prix forfaitaire et gestion à compensation forfaitaire.

Les coûts d'exploitation des opérateurs de transport urbain constituent actuellement une préoccupation fondamentale pour les responsables politiques des différentes collectivités locales. Le secteur est en effet perpétuellement déficitaire. Afin d'assurer au transport son caractère de service public, le niveau moyen des prix ne garantit pas l'équilibre financier de l'exploitation. Les élus locaux se trouvent dans l'obligation de réaliser un compromis entre d'une part la bonne santé financière de l'exploitation assurée par le versement de subventions ${ }^{3}$ et d'autre part la mission de service public du transport local.

Dans cette étude, nous modélisons la structure de la production de l'industrie du transport urbain Français à l'aide d'une fonction de coût de type translog et d'une base de données constituée d'observations portant sur 60 opérateurs au cours de la période 19851993. Cette modélisation nous permet tout d'abord de mettre en évidence le caractère déficitaire de l'industrie à partir de l'estimation des coûts marginaux et moyens des exploitants et de leur comparaison avec le prix moyen du service. Les résultats suggèrent que le prix moyen pratiqué est en moyenne supérieur au coût marginal mais inférieur de moitié au coût variable moyen. Comme il a été suggéré, l'environnement réglementaire définit différents types de risques financiers encourus par les opérateurs et ainsi différentes incitations en matière de réduction des coûts. L'impact des schémas réglementaires sur la formation des coûts est un des thèmes majeurs de la nouvelle théorie de la réglementation des monopoles (voir notamment Laffont et Tirole [1993] et Laffont [1994]). La recherche empirique a cependant peu traité ce problème. Mathios et Rogers [1989] et Dalen et Gomez-Lobo [1995] ont utilisé des modèles à formes réduites pour étudier les effets de différents modes réglementaires sur les prix ou les coûts d'exploitation.

Dans le cadre de l'industrie du transport urbain proprement français, Caillaud et Quinet [1993] ont tenté de modéliser le choix du mode de réglementation comme une variable endogène fonction de plusieurs paramètres comme l'aversion au risque de l'A.O. et de l'exploitant, l'importance de la volatilité des recettes et des coûts etc. Croissant [1996,1997] a estimé les coûts d'exploitation des opérateurs et la fonction de demande des consommateurs. Wunsch [1996] et Kerstens [1996] proposent une estimation des inefficacités productives des opérateurs. Aucune approche empirique n'a cependant, à notre connaissance, considéré l'influence des schémas incitatifs. C'est ce que nous essayons de réaliser dans cet article à travers une approche très simple. Les résultats confirment les intuitions de départ. Les opérateurs qui sont réglementés par des schémas moins incitatifs ont des coûts d'exploitation plus élévés. 
La section 2 expose l'environnement réglementaire de l'industrie et rappelle de manière succincte l'intérêt d'un modèle économétrique à forme réduite. Une analyse descriptive de la base de données et une présentation de la construction des variables sont présentées dans la section 3. Les caractéristiques de la forme translog et la structure stochastique du modèle sont proposées dans la section 4. Les résultats sont mis en évidence dans les sections 5. La section 6 conclut et avance les perspectives suggérées par l'étude.

\section{Réglementation des transports urbains et rappels empiriques}

\subsection{L'environnement institutionnel et réglementaire}

La sphère des transports urbains est soumise à des règles institutionnelles et réglementaires particulières. Il s'agit d'un service public dont la politique est déterminée par une personne publique, l'Autorité Organisatrice ${ }^{4}$, tandis que l'exploitation est en général confiée à une entreprise privée, l'exploitant. Les relations entre les deux parties sont régies par une convention qui définit le type de réglementation adopté. L'objectif de l'A.O. est d'inciter l'exploitant à satisfaire certaines obligations économiques et sociales choisies par elle, alors que les propres intérêts de l'entreprise et son appartenance à des groupes importants la conduiraient à poursuivre d'autres objectifs 5 .

L'instauration de la Loi d'Orientation des Transports Intérieurs (LOTI) en 1982 est issue d'une volonté générale de réglementation du droit des transports. Divers principes en sont issus, notamment l'organisation de la politique de transport par les collectivités locales, la définition du périmètre de transport $^{6}$, le financement par les bénéficiaires directs et autres bénéficiaires privés ou publics indirects etc. En 1993, la loi Sapin, surnommée loi anti-corruption, impose des contraintes importantes à l'A.O. en matière de transparence dans les choix de l'Exploitant à l'issue de chaque convention.

Les deux parties sont liées par un contrat qui précise, entre autres, la consistance générale des services à fournir par l'exploitant, les conditions de l'exploitation du service, la nature du financement et les modalités de contrôle de l'utilisation des fonds engagés. Le point primordial de l'analyse est la nature du financement : Le transport urbain fait partie de la sphère du service public. Plus qu'un mode de déplacement, il est devenu un véritable outil pour la formation du paysage économique et social urbain. La collectivité locale, responsable de l'organisation du transport sur son territoire, rend directement des comptes à ses électeurs qui sont également les usagers du réseau. Aussi, sa préoccupation principale est la maximisation du bien-être social. Pour cela, le prix du bien transport imposé par l'A.O. est bien inférieur au prix qui permettrait d'assurer l'équilibre financier de l'exploitation qui est déficitaire en permanence ${ }^{7}$. Le versement d'une subvention ${ }^{8}$ à l'A.O. est donc nécessaire pour assurer l'équilibre de l'exploitation. Toutefois, la subvention ne constitue pas l'essentiel du financement des exploitations, puisque ce dernier dépend de la forme contractuelle qui lie les deux parties.

\subsection{Formes contractuelles}


Trois grands types de contrats, qui diffèrent par la propriété finale des recettes commerciales et la nature du remboursement des coûts d'exploitation, sont utilisés ${ }^{9}$. Le premier type, intitulé Contrat de gérance, définit une convention où l'exploitant, à l'issue de l'exercice ${ }^{10}$, cède les recettes commerciales à l'A.O. qui lui rembourse en contrepartie la totalité de ses coûts ex-post. L'exploitant ne supporte donc aucun risque. Dans le cas des contrats de gestion avec compensation forfaitaire, l'exploitant assure le risque sur les recettes ${ }^{11}$ et les charges et reçoit une subvention du type de celle définit précédemment pour assurer l'équilibre du budget prévisionnel. Dans le dernier cas, celui des contrats de gestion à prix forfaitaires, l'exploitant ne conserve que le surplus des recettes $^{12}$ lorsque le niveau effectif dépasse le niveau prévu ex-ante et obtient un remboursement forfaitaire de ses coûts fixé ex-ante.

Il est aisément compréhensible que ces différents contrats génèrent des incitations diverses en terme de réduction des coûts d'exploitation. Les régimes de type compensation forfaitaire et prix forfaitaire sont toutefois identiques en terme d'incitation puisque les deux procédés qui consistent à rembourser les coûts prévisionnels et recevoir les recettes prévisionnelles ou verser une rémunération susceptible d'équilibrer le budget prévisionnel sont similaires. Ces deux types de mécanismes s'apparentent en fait à une réglementation de type prix-fixe tandis que les régimes de type gérance s'apparentent plutôt à une réglementation de type coût $d u$ service.

Dans l'industrie du transport urbain, la productivité des opérateurs est fortement affectée par les problèmes de congestion du trafic sans cesse croissants. Généralement, lorsqu'un véhicule concède un retard important sur un trajet, l'opérateur incorpore au réseau déjà en place un véhicule supplémentaire qui assure le service à la place du véhicule retardé. Ainsi, pour assurer un niveau de service donné, un opérateur peu efficace et/ou qui fournit un niveau d'effort de productivité peu élevé utilise une quantité de facteurs de production plus élevée à niveau de demande donné d'où des coûts d'exploitation plus importants.

Actuellement, les collectivités locales subissent d'importantes difficultés financières et la dérive des coûts des exploitants de transport urbain accentue leur endettement, notamment dans le cas des contrats de Gérance (voir Domenach [1987]). Aussi, les Autorités Organisatrices ont intérêt à inciter l'exploitant à améliorer sa productivité. Mais elles doivent aussi se doter d'une capacité d'expertise pour le suivi et le contrôle de l'exploitation afin de s'assurer que la définition des services rendus est adaptée à la demande et conforme aux prévisions budgétaires. Or, il s'avère que la capacité d'expertise de l'A.O. est difficilement crédible, les responsables des collectivités locales ne détenant pas toujours l'information technique propre au monde des transports nécessaire à un contrôle de gestion fiable. Selon Domenach [1987] :

"Il est impossible de déterminer, dans l'absolu, quel est le nombre de bus nécessaire sur un réseau. Il en va de même pour la consommation de gazole qui varie pour un même véhicule d'un chauffeur à l'autre, suivant le type de conduite ou de réglage etc. [...]. On a trop souvent l'impression que les services transports des collectivités sont composés de généralistes au lieu de professionnels qualifiés capables de faire le poids face aux techniciens "pointus » des entreprises exploitantes. [...] Tout cela peut expliquer une tendance à un certain laxisme qui ne permet pas de créer le rapport de compétence souhaitable pour la négociation des contrats sur des bases égalitaires entre les parties ». 
Cette citation de Domenach est évidemment ancienne et on peut imaginer qu'un progrès non négligeable a été effectué depuis. La publication annuelle de statistiques comme celles utilisées dans cette étude permet notamment aux autorités d'user du principe de la "Yardstick competition". Il reste néanmoins qu'un déséquilibre subsiste en terme de connaissances techniques au moment des négociations entre les deux parties.

La situation d'asymétrie d'information entre principaux et agents ne doit donc pas être rejetée mais ne sera pas abordée ici. Toutefois, nous tentons de montrer que les deux types de régimes définis précédemment ont des effets différents en terme d'incitation à la productivité. La conjecture, qui fait l'objet du test empirique ci-dessous, est qu'un exploitant à qui l'A.O. rembourse intégralement les coûts ex-post n'a pas la même incitation à les réduire qu'un exploitant qui prend tous les risques industriels.

\subsection{L'utilisation des formes réduites}

Un modèle à forme réduite peut fournir une intuition sur les effets de certaines variables entre elles sans nécessiter une modélisation complète de l'environnement qui caractérise l'activité de production. Dans le cadre particulier du transport urbain, Dalen et Gomez-Lobo [1995] se sont servis de tels modèles pour mesurer les effets contractuels sur les coûts d'exploitation dans l'industrie norvégienne. ${ }^{13}$

L'industrie du transport urbain Norvégien est régulée comme la plupart des industries de transport locales Européennes. Le caractère déficitaire de l'opération nécessite le versement de transferts importants. Le caractère coûteux de l'utilisation des fonds publics a motivé un bouleversement important de la régulation du secteur. En 1983, la règle du remboursement intégral du déficit a laissé la place à des procédures de négociations des objectifs financiers en début d'exercice. Au départ, les négociations sur les prix et les coûts étaient individuelles. En 1986, plusieurs autorités locales adoptèrent un système standard qui imposa notamment un certain nombre de critères universels pour la détermination des coûts d'exploitation. Ce procédé s'est avéré beaucoup plus incitatif en matière de réduction des coûts que celui basé sur la négociation individuelle. Dalen et Gomez Lobo observent l'impact d'un mode particulier de régulation sur les coûts d'exploitation. Ils utilisent pour cela une forme fonctionnelle de type translog et incorporent au modèle une variable muette qui prend la valeur 1 si l'opérateur est régulé selon un mode standard. L'estimation suggère que les entreprises régulées sur la base d'un schéma standard ont en moyenne des coûts inférieurs de 3.6\% par rapport aux entreprises régulées sur la base de critères individuels. Ceci tend à montrer que ces dernières sont moins incitées à la réduction de leurs coûts d'exploitation.

L'approche considérée dans cette étude est proche de celle de Dalen et GomezLobo [1995]. Une forme réduite de type translog sert de support à l'estimation des coûts d'exploitation. Une variable, qui caractérise le type de schémas réglementaire en activité, est introduite pour étudier l'effet de celui-ci sur les coûts. La section suivante fait l'objet d'une présentation des données qui serviront à l'estimation de la fonction de coût.

\section{Les données}

Nous disposons d'observations sur 60 exploitants de réseaux urbains ${ }^{14}$ pour la période 1985-1993. La grande diversité des variables disponibles ${ }^{15}$ permet une étude 
empirique assez vaste qui ne peut se réduire à celle présentée dans cet article. Ces données sont éditées annuellement par le $\mathrm{CERTU}^{16}$ et le GART ${ }^{17}$. Pour l'estimation de la fonction de coût de l'exploitant, il est nécessaire de définir plusieurs variables dont nous exposons la construction dans cette section.

On considère que le processus de production nécessite trois facteurs de production fondamentaux, le travail, l'énergie et les véhicules de transport. Dans $90 \%$ des cas, le capital roulant est détenu par l'A.O. Ainsi, en cas de séparation de l'A.O. et de l'exploitant, l'A.O. conserve le capital. Il arrive cependant que l'exploitant achète l'ensemble du capital au début de l'exercice et en devienne propriétaire pendant toute la durée de la convention avant de le revendre en fin d'exercice. Ce cas est cependant peu fréquent. En définitive, nous faisons l'hypothèse suivante

\section{HYPOTHESE 1. L'infrastructure et l'ensemble du parc des véhicules sont détenus par l'A.O.}

Cette règle évite à l'opérateur de supporter le poids des charges liées au capital. Nous considérons alors deux grandes catégories de charges annuelles pour l'opérateur, celles liées à la rémunération de la main d'oeuvre et celles liées à la consommation de l'énergie. La main d'oeuvre comprend les chauffeurs des véhicules et le personnel administratif. Nous ne disposons malheureusement que d'un chiffre brut pour la masse salariale qui ne permet pas de distinguer les deux catégories de salariés. Afin d'obtenir un prix moyen du travail, nous divisons le volume total des salaires par l'équivalent personnes-année employé dans l'entreprise ${ }^{18}$. Le prix moyen de l'énergie résulte de la division des charges correspondantes par la quantité de fuel utilisée sur une année. Les prix du travail et de l'énergie sont représentés respectivement par $p_{L}$ et $p_{M}$.

Le niveau de production, noté $y$, est représenté par la variable véhiculeskilomètres offerts. ${ }^{19}$ Elle rend à la fois compte de la capacité effective moyenne utilisée pour assurer le service et de la distance kilométrique parcourue par tous les véhicules sur l'année. Le transport est couramment assuré par différents modes de transport, à savoir le bus, le métro ou le VAL. Chacun de ces modes est confronté au effets externes liés au trafic à des degrés divers et donc s'avère différemment coûteux en terme d'exploitation. Comme Wunsch [1996] l'a suggéré dans son étude sur les réseaux européens, il conviendrait de distinguer ces différents modes aux moments de la spécification des coûts. Cette étude ne considère pas une telle distinction. D'une part, Paris et Lyon ne font pas partie de la base de donnée, la part du métro et du VAL dans l'offre de transport considérée devient alors négligeable au cours de la période considérée. D'autre part, les données disponibles pour ces modes sembles peu fiables.

Le capital alloué par l'Autorité Organisatrice, dénoté $k$, est défini comme la capacité totale disponible du réseau en nombre de places. La variable INF rend compte de l'infrastructure disponible. Il s'agit de la taille du réseau en nombre de kilomètres.

La vitesse commerciale ${ }^{20}$ du réseau est une variable tout à fait pertinente pour décrire la qualité du service car elle détermine directement la durée d'un trajet entre un point de départ et un point d'arrivée. Une telle variable est assez particulière car elle influe simultanément sur la demande des consommateurs et sur la formation des coûts d'exploitation. Le temps de déplacement pour l'usager est un critère essentiel du choix du mode de transport. Il préfère utiliser son véhicule personnel s'il juge qu'il constitue 
un moyen de locomotion plus rapide. Ainsi, une augmentation de la demande de transport urbain passe avant tout par une amélioration de la vitesse commerciale. En parallèle, Un même trajet parcouru à vitesse plus élevée, donc en moins de temps, nécessite un investissement moindre en matériel roulant et en main d'oeuvre sur l'année. Ainsi, une amélioration de la vitesse moyenne du réseau permet une réduction non négligeable des coûts d'exploitation ${ }^{21}$. C'est pourquoi la vitesse moyenne du réseau, qui chute régulièrement chaque année en raison de l'augmentation du volume de la circulation générale, constitue l'enjeu majeur actuel de toutes les compagnies de transport et Autorités Organisatrices. La variable VIT donne la valeur de la vitesse commerciale annuelle pour chaque réseau.

Nous introduisons une variable temporelle afin de mettre en évidence l'existence d'un éventuel progrès technique d'une année à l'autre. La variable $P F$ prend la valeur 1 si le contrat est de type prix-fixe, $O$ sinon. Notons que

HYPOTHESE 2 . Le type de contrat considéré dans cette étude est supposé exogène.

Cette hypothèse est naturellement discutable. En effet, bien que l'autorité dispose en théorie du pouvoir de décision final, le choix du mode de régulation doit faire l'objet de nombreuses négociations. On peut par exemple imaginer que l'opérateur préfère un contrat de type coût du service s'il éprouve de l'aversion au risque ou si la demande de transport est incertaine. ${ }^{22}$ De la même façon, le régulateur peut vouloir mettre en place un schémas de type prix-fixe si les coûts d'exploitation de la période précédente sont élevés. De là, il faudrait introduire un effet rétroactif entre les coûts et le mode de régulation.

\section{TABLEAU 1}

Cette étude se contente d'observer les effets des régimes réglementaires sur les coûts lorsque ces régimes sont en place. La possibilité d'une approche structurelle et/ou dynamique, plus propice à un modèle de choix de contrats, est évoquée en conclusion et sera traitée, dans tous les cas, au cours d'une étude ultérieure.

La Table 1 présente quelques statistiques descriptives de la base de donnée. A la lecture de cette table, il apparaît que la masse salariale représente près de $90 \%$ des coûts totaux. Il est vraisemblable qu'à l'intérieur même de cette masse salariale, celle allouée aux conducteurs est la plus importante ${ }^{23}$. La base de données contient 540 observations. Parmi elles, nous dénombrons 236 contrats de type gérance (43.7\%), 116 contrats de type prix forfaitaire $(21.5 \%)$ et 115 contrats de type compensation forfaitaire $(21.3 \%)^{24}$.

\section{Le modèle économétrique}

\subsection{La fonction translog}

La théorie de la dualité stipule que le processus de production d'une firme peut être indifféremment représenté par une fonction de production

$$
Y=f(x, t),
$$

ou une fonction de coût concave et homogène de degré 1 par rapport aux prix 


$$
C=h(y, p, t),
$$

où $x, p$ sont deux vecteurs indiquant respectivement la quantité et le prix des facteurs de production utilisés par le processus et $t$ est un indicateur temporel. Pour la validation de cette théorie, il est nécessaire que les prix des facteurs de production soient exogènes pour la firme et que cette même firme adopte un comportement de minimisation de ses coûts. Le choix de l'une ou l'autre des deux formes dépend en fait du caractère exogène ou endogène de la variable indiquant le niveau de production $y$. Dans notre cas, ce niveau de production est exogène pour la firme, donc nous estimons une fonction du type de celle définie dans (2).

Comme il a été spécifié dans la précédente section, le capital, c'est à dire l'infrastructure et l'ensemble du parc des véhicules, est détenu par l'Autorité Organisatrice. Ainsi, l'ensemble des charges liées à l'entretien ou au renouvellement du capital est entièrement supporté par le principal. Chaque firme minimise donc le coût d'un sous ensemble de facteurs de production, le travail et l'énergie, conditionnellement au niveau du capital mis à sa disposition par l'autorité. Le capital peut alors être considéré comme un facteur de production quasi-fixe et la fonction de coût considérée n'est alors plus une fonction de coût total mais une fonction de coût variable. Une telle approche permet de considérer que l'industrie du transport urbain est à chaque période en déséquilibre par rapport à la capacité disponible concédée par l'Autorité Organisatrice.

Afin de décrire les structures productives de l'industrie du transport urbain, nous introduisons la fonction de coût variable suivante :

$$
C V=g(y, p, k, t, z, v)
$$

où $C V$ désigne le coût variable, $p$ est un vecteur du prix des facteurs, $k$ est le capital alloué par l'Autorité Organisatrice, $t$ un trend, $z$ rend compte de la taille du réseau et $v$ englobe plusieurs variables de contrôle qui rendent compte de certaines caractéristiques qualitatives du réseau et de l'environnement réglementaire. De manière à effectuer une distinction entre la taille du réseau ${ }^{25}$ et le niveau de l'offre proprement dite, nous introduisons dans cette fonction une variable qui rend compte de la longueur cumulée des lignes de chaque réseau. L'approche considère en quelque sorte chaque exploitant comme une firme multi-produits ${ }^{26}$ qui offre à la fois une quantité de véhiculeskilomètres et une taille de réseau particulière. Dans le cadre particulier de la fonction translog spécifiée ci-après, cette approche conduit à estimer un certain nombre de termes de second degré puisqu'il est nécessaire de croiser les deux variables rendant compte de la production entre elles et avec les variables caractérisant les prix des facteurs et le capital.

Nous supposons que les coûts d'exploitation peuvent être caractérisés par une fonction de coût de type translog telle que définie par Christensen et al. [1973]. La forme translog est dite flexible, elle fournit une approximation de second ordre d'une fonction de coût dont la structure est a priori inconnue.

HYPOTHESE 3 : La fonction de coût variable est représentée par une forme translog.

Considérons la fonction de coût Translog 


$$
\begin{aligned}
\ln C V=\alpha_{0} & +\sum_{i} \alpha_{i} \ln p_{i}++\alpha_{y} \ln y+\alpha_{t} t+\alpha_{k} \ln k+\alpha_{I} \ln I N F+\alpha_{V I T} \ln V I T+\alpha_{P F} P F \\
& +\sum_{\mathrm{i}} \alpha_{i y} \ln p_{i} \ln y+\sum_{\mathrm{i}} \alpha_{i k} \ln p_{i} \ln k+\sum_{\mathrm{i}} \alpha_{i I} \ln p_{i} \ln I N F+\frac{1}{2} \alpha_{y y}(\ln y)^{2} \\
& +\frac{1}{2} \alpha_{k k}(\ln k)^{2}+\frac{1}{2} \alpha_{I I}(\ln I N F)^{2}+\alpha_{y k} \ln y \ln k+\alpha_{y I} \ln y \ln I N F \\
& +\frac{1}{2} \sum_{i} \sum_{j} \alpha_{i j} \ln p_{i} \ln p_{j}+\alpha_{k I} \ln k \ln I N F+\psi_{C}, \quad i \in\{\mathrm{L}, \mathrm{M}\} .
\end{aligned}
$$

où $C V$ est le coût supporté par l'exploitant, $p_{i}$ le prix du facteur $i, I N F$ dénote la taille du réseau et $\psi_{c}$ la structure stochastique discutée ci-dessous.

Pour que la fonction (4) soit une fonction de coût, il est nécessaire qu'elle soit concave $^{27}$, homogène de degré 1 par rapport aux prix et que $\alpha_{i j}=\alpha_{j i}$. L'homogénéité de degré 1 par rapport aux prix est induite par la condition

$\sum_{i} \alpha_{i}=1, \quad \sum_{\mathrm{i}} \alpha_{i y}=0, \sum_{\mathrm{i}} \alpha_{i k}=0, \sum_{\mathrm{i}} \alpha_{i I}=0, \sum_{\mathrm{i}} \alpha_{i j}=\sum_{\mathrm{j}} \alpha_{i j}=0, \quad i \in\{\mathrm{L}, \mathbf{M}\}$.

Notons que la fonction est de type Cobb-Douglas si

$$
\alpha_{i j}=\alpha_{y y}=\alpha_{k k}=\alpha_{I I}=\alpha_{i y}=\alpha_{i k}=\alpha_{i I}=\alpha_{y k}=\alpha_{y I}=\alpha_{k I}=0 \quad \forall i, j \in\{\mathrm{L}, \mathrm{M}\} .
$$

D'après le Lemme de Shephard, nous pouvons dériver l'expression (4) par rapport aux prix des facteurs de production afin d'obtenir la part de coût de chaque facteur dans le coût total:

$$
\frac{\partial \ln C V}{\partial \ln p_{i}}=\frac{p_{i} x_{i}}{C V}=S_{i}=\alpha_{i}+\sum_{j} \alpha_{i j} \ln p_{j}+\alpha_{i y} \ln y+\alpha_{i k} \ln k+\alpha_{i I} \ln I+\psi_{s_{i}}, \forall i, j \in\{\mathrm{L}, \mathrm{M}\}
$$

où $x_{i}$ est la quantité du facteur $i$ utilisée dans le processus de production et $\psi_{s_{i}}$ la structure stochastique de la fonction de part de coût $i$ discutée ci-dessous. L'estimation conjointe de (4) et (7) est nécessaire en raison du grand nombre de paramètres à estimer.

\subsection{Spécification de la structure stochastique.}

La méthode d'estimation utilisée est le maximum de vraisemblance. Comme la fonction de vraisemblance est connue (Voir Magnus [1982]), nous nous contentons de spécifier ici la structure stochastique du modèle.

Face au nombre important de paramètres à estimer, il est utile, voire nécessaire de considérer simultanément la fonction Translog et les fonctions de part de coût. La somme des parts étant égale à un, une des équations de part est délaissée afin d'éviter tout problème de singularité de la matrice des variances-covariances des résidus. Le choix de l'équation à abandonner n'a aucune incidence sur l'estimation des paramètres 
(Voir Berndt [1990], par exemple). Considérons une base de données constituée de $n$ firmes observées sur $T$ années. En raison de la linéarité de la fonction translog, définissons un système de deux équations ${ }^{28}$ :

$$
Y_{i t}=X_{i t} \beta+\psi_{i t} \quad i=1,2, \ldots n, \quad t=1,2, \ldots T,
$$

où $\psi_{i t}=\left(\psi_{c, i t} \psi_{S_{i}, i t}\right)^{\prime}, Y_{i t}, \psi_{i t}$ sont des vecteurs de taille $(2,1)$ et $X_{i t}$ est une matrice de taille $(2, k)$. Nous introduisons un effet aléatoire spécifique aux firmes $\xi_{i}$ et nous supposons :

HYPOTHESE 3 : La structure stochastique des résidus est définie par $\psi_{i t}=\xi_{i}+\omega_{i t}$.

Les erreurs $\xi_{i}$ et $\omega_{i t}$ sont distribuées indépendamment selon une loi normale de moyenne nulle et de matrice de variances-covariances $\Psi$ et $\Omega$ respectivement. Les matrices $\Psi$ et $\Omega$ sont d'ordre 2. L'intérêt de cette spécification est de supposer une corrélation nulle entre les firmes mais une corrélation non nulle dans le temps pour chaque firme. Ainsi,

$$
\begin{aligned}
E\left(\psi_{i t} \psi_{j s}^{\prime}\right) & =\Omega+\Psi \text { si } i=j \text { et } t=s, \\
& =\Psi \quad \text { si } i=j \text { et } t \neq s, \\
& =0 \quad \text { si } i \neq j .
\end{aligned}
$$

La matrice de variances-covariances des $\psi_{i t}$ de taille $(n, n)$ est

$$
\Gamma=E\left(\psi_{i t} \psi_{j s}^{\prime}\right)=\left[\begin{array}{ccc}
\Sigma & 0 & 0 \\
0 & \ddots & 0 \\
0 & 0 & \Sigma
\end{array}\right],
$$

où $\Sigma$, de taille $(T, T)$ est la matrice

$$
\Sigma=\left[\begin{array}{cccc}
\Omega+\Psi & \Psi & \ldots & \Psi \\
\Psi & \Omega+\Psi & \ldots & \Psi \\
\vdots & \vdots & \ddots & \vdots \\
\Psi & \Psi & \ldots & \Omega+\Psi
\end{array}\right] .
$$

Une fois définie la fonction de coût et sa structure stochastique, nous pouvons étudier les caractéristiques de la structure productive du transport urbain et les effets des structures contractuelles sur les coûts de production.

\section{Structures productives de l'industrie du transport urbain et effets des schémas réglementaires}

\subsection{Structures productives}

L'ensemble des résultats est exposé en page 14. La Table 2 présente les résultats de l'estimation du modèle. La table 3 donne une estimation des parts des facteurs de production dans le coût variable total pour une firme moyenne. La fonction de coût 
estimée est concave au point moyen de l'échantillon. Les élasticités des coûts par rapport aux prix des facteurs de production sont proches des parts moyennes de chaque facteur dans le coût variable d'exploitation.

La variable VIT a un coefficient négatif, ce qui implique qu'une amélioration de la vitesse commerciale réduit les coûts de l'exploitant. Cette amélioration passe par une meilleure gestion du trafic urbain, comme l'implantation de voies réservées, de sites propres ou de feux de signalisation spécifiques entre autres. Or cette gestion dépend des collectivités locales responsables de l'aménagement du territoire, soit de l'A.O., l'exploitant n'ayant pas d'influence directe. L'intérêt de l'amélioration de la vitesse moyenne du réseau est double pour l'A.O. puisqu'elle fait croître la demande de transport urbain et réduit aussi les coûts de l'exploitant. Son incitation à faciliter la fluidité du trafic n'est donc pas nulle mais dépend du compromis entre les coûts engendrés par l'aménagement de l'infrastructure et la réduction des coûts d'exploitation.

L'élasticité des coûts par rapport au capital est négative, ce qui tend à montrer que l'augmentation de la capacité du réseau réduit les coûts variables de l'exploitant. Le modèle présente un progrès technique significatif puisque le coefficient de la variable $t$ est négatif.

Rappelons que notre base de données est constituée de données de Panel, c'est à dire que l'observation des firmes s'effectue sur plusieurs périodes. Ainsi, il est possible d'observer l'effet du temps sur les coûts de production. En effet, sachant que la durée d'observation dans notre cas est de neuf ans, l'effet des progrès techniques et scientifiques voire d'organisation n'est pas négligeable. Toutes choses égales par ailleurs, nous pouvons nous attendre à une diminution des coûts dans le temps. Le taux de progrès technologique annuel est donné par la relation (voir Greene [1983]).

$$
\delta=-\frac{\partial \ln C}{\partial t}=-\alpha_{t}
$$

D'après l'estimation, il apparaît que la diminution annuelle moyenne des coûts induite par le progrès est égale à $0.62 \%$ des coûts totaux.

A partir de l'estimation présentée, nous calculons les élasticités prix propres et croisées de la demande de facteurs de production. Les élasticités sont données par l'expression

$$
\begin{aligned}
& \varepsilon_{i j}=\frac{\alpha_{i j}+S_{i} S_{j}}{S_{i}}, \quad i \neq j, \\
& \varepsilon_{i i}=\frac{\alpha_{i j}+S_{i}^{2}-S_{i}}{S_{i}},
\end{aligned}
$$

où $\varepsilon_{i i}$ est l'élasticité-prix propre de la demande hicksienne en bien $i$. Elle indique comment évolue la demande de facteur de production $i$ lorsque le prix de ce facteur augmente ou diminue. Pour les élasticités prix croisées, nous utilisons l'élasticité de substitution de Morishima ${ }^{29}$ :

$$
M_{i j}=\varepsilon_{j i}-\varepsilon_{i i},
$$

où $M_{i j}$ est l'élasticité-prix croisée de la demande de bien $i$. Elle indique comment évolue la demande de facteur de production $i$ lorsque le prix d'un autre facteur augmente ou diminue. La table 4 présente les élasticités prix propres et croisées des 
demandes de facteurs. Les élasticités prix croisées sont positives, les facteurs de production entrant dans les coûts variables sont donc a priori substituables. Les estimations des élasticités prix propres indiquent que le travail est le facteur de production le moins sensible à une augmentation de son prix propre. Il s'apparente à un facteur quasi-fixe.

\section{2: Coûts marginaux et coûts variables moyens.}

A partir de la fonction de coût translog définie dans (4), nous dérivons

$$
C V(y)=A y^{g(y)},
$$

avec

$$
A=p_{M} e^{\alpha_{0}+\alpha_{t} t+\alpha_{v i t} \ln v i t+\alpha_{P F} P F}\left(\frac{p_{L}}{p_{M}}\right)^{\alpha_{L}+\frac{1}{2} \alpha_{L L} \ln \left(\frac{p_{L}}{p_{M}}\right)} k^{\alpha_{k}+\frac{1}{2} \alpha_{k k} \ln k+\alpha_{k L} \ln \left(\frac{p_{L}}{p_{M}}\right)} I N F^{\alpha_{I}+\frac{1}{2} \alpha_{I I} \ln I N F+\alpha_{L L} \ln \left(\frac{p_{L}}{p_{M}}\right)+\alpha_{l k} \ln k}
$$

et

$$
g(y)=\alpha_{y}+\frac{1}{2} \alpha_{y y} \ln y+\alpha_{L y} \ln \frac{p_{L}}{p_{M}}+\alpha_{I y} \ln I N F+\alpha_{k y} \ln k
$$

où

$$
\frac{d g(y)}{d y}<0 \text { et } \frac{d^{2} g(y)}{d y^{2}}>0 .
$$

Le prix des facteurs est fixe. A chaque période, la fonction de coût dépend donc uniquement du volume de production y et de la quantité de capital définie par l'Autorité Organisatrice. A partir de l'expression (16), nous calculons le coût marginal induit par l'offre d'un véhicule-kilomètre supplémentaire à l'aide de l'expression

$$
\frac{\partial C V}{\partial y}=A\left(g_{y}(y) \ln y+g(y) y^{-1}\right) e^{g(y) \ln y} .
$$

Le coût marginal ainsi défini donne le coût supplémentaire induit par l'offre additionnelle d'un unique véhicule-kilomètre au cours de l'année. Pour permettre une comparaison pertinente avec le prix moyen du transport imposé par l'opérateur, le coût marginal ainsi calculé peut être rapporté au coût marginal issu du transport d'un voyageur supplémentaire. ${ }^{30}$ Les valeurs du prix moyen calculé et du coût marginal estimé pour un opérateur moyen au cours de la période sont présentées dans la table 5. Si les valeurs pour un opérateur moyen suggèrent un prix pratiqué supérieur au coût marginal moyen, les comparaisons individuelles pour chaque entreprise évoquées dans les figures 1 et 2 ci-dessous montrent que certaines entreprises font face à un coût marginal supérieur au prix moyen.

Actuellement, la structure de la tarification en place ne reflète pas les fluctuations quotidiennes du vrai coût marginal lié à l'offre de transport. Ainsi, elle ne considère pas les périodes de pointe ou la distance des trajets. La structure tarifaire en place est uniforme par rapport au moment de la journée, à la distance parcourue et au 
mode de service. En 1994, 75\% des entreprises de transport pratiquent le tarif unique, $20 \%$ le tarif par zone et $5 \%$ le tarif par section. ${ }^{31}$ Devant le déficit permanent de l'industrie du transport public, on peut se demander pourquoi une telle structure tarifaire est maintenue. Plusieurs suggestions peuvent être avancées pour justifier ce principe : Une structure tarifaire uniforme évite aux firmes et aux consommateurs de s'engager dans des activités de collecte d'information qui peuvent s'avérer coûteuses. Par exemple, une bonne connaissance de l'élasticité instantannée de la demande, nécesssaire pour une tarification appropriée, n'est pas forcément disponible. Plus simplement, une structure tarifaire parfaitement adaptée aux fluctuations des coûts marginaux de manière continue semble difficilement réalisable ou obligerait les consommateurs à supporter une incertitude élevée.

Le coût variable moyen est le coût variable par unité produite. D'après (16), (17) et (18), nous obtenons

$$
C V M=\frac{C(y)}{y}=A y^{g(y)-1} .
$$

\section{TABLEAU 2}

\section{TABLEAU 3}

\section{TABLEAU 4}

\section{TABLEAU 5}

\section{GRAPHIQUE 1}

\section{GRAPHIQUE 2}

Afin de permettre une comparaison pertinente avec les prix pratiqués, à savoir les recettes moyennes, le coût variable moyen peut être ramené au coût variable moyen d'un voyageur transporté. ${ }^{32}$ La table 5 en donne la valeur estimée pour une entreprise moyenne au cours de la période. Il apparaît que le coût moyen estimé est environ deux fois supérieur à la recette moyenne calculée. L'examen des figures 1 et 2 permet de tirer un certain nombre d'enseignements propres au cadre de l'industrie du transport urbain. Ces figures donnent une représentation graphique des coûts variables estimés et revenus moyens et des coûts marginaux estimés pour chaque firme pour l'année 1985 et la période 1985-1993.

Chaque niveau de production représente une firme particulière de l'échantillon. Il apparaît tout d'abord que les coûts variables moyens et marginaux et le revenu moyen sont des fonctions décroissantes du nombre de voyageurs transportés. Ainsi les compagnies les plus petites en terme d'offre ont les coûts variables moyens les plus élevés. En moyenne, les coûts variables moyens sont deux fois supérieurs aux recettes moyennes, ce qui explique le caractère déficitaire de l'industrie. Les frais variables et les 
frais fixes ne sont pas couverts, les entreprises produisent à perte car les prix proposés par les Autorités Organisatrices sont inférieurs de moitié au niveau qui permettrait d'équilibrer les budgets d'exploitation. L'examen de la figure 1 montre que l'écart (en valeur absolue au moins) entre coûts variables et recettes moyens se restreint lorsque le niveau de production est plus élevé. Cette dernière remarque est un argument potentiel pour le maintient du statut monopolistique des entreprises de transport urbain.

\subsection{Effets des mécanismes réglementaires sur les coûts.}

A partir des approches économique et économétrique présentées dans la section 4, nous avons montré jusqu'à présent comment les coûts d'exploitation des opérateurs de transport urbain pouvaient être affectés par la vitesse commerciale du réseau ou le progrès technique.

A cette première catégorie d'effets s'ajoutent également les effets liés à l'environnement contractuel qui réglemente les décisions des opérateurs de transport urbain. Ces effets sont endogènes dans le sens où l'environnement réglementaire définit l'effort de productivité des opérateurs et donc leurs coûts. Nous avons montré dans la section 2 qu'en matière d'incitation à la productivité, deux schémas incitatifs s'opposent, d'une part les contrats de type prix-fixe, d'autre part les contrats de type coût du service. L'objet de cette étude est de montrer que les deux types de contrat ont des effets opposés sur la formation des coûts.

Dans ce but, nous introduisons la variable $P F$ dans la fonction translog présentée en (4). Rappelons que cette variable prend la valeur 1 si le contrat est de type gestion à prix forfaitaire ou gestion à compensation forfaitaire et $O$ sinon. Parce que les contrats de type prix-fixes sont plus incitatifs en matière d'effort de productivité, la variable $P F$ est supposée avoir un effet négatif sur les coûts ${ }^{33}$.

La table 2 présente également les effets de l'introduction de la variable $P F$, sur la formation du coût. La variable est significative et son coefficient est négatif. Ce résultat indique que les firmes réglementées par des contrats de type prix-fixe ont en moyenne des coûts inférieurs de $2.05 \%$ par rapport aux firmes réglementées par des schémas de type coût $d u$ service. Ces résultats confirment les hypothèses de départ qui stipulaient qu'une entreprise de transport a une incitation à réduire ses coûts qui dépend du type de contrat que l'Autorité Organisatrice lui propose. En l'occurrence, il n'est apparemment pas optimal pour l'A.O. de proposer un contrat qui spécifie que l'intégralité des coûts de l'exploitant lui sera remboursée ex-post.

\section{Conclusion}

A la lumière des résultats présentés dans cet article, nous pouvons tirer plusieurs remarques sur les structures productives de l'industrie du transport urbain en France. Parce que l'industrie est soumise à des effets externes négatifs et à une réglementation aux effets incitatifs contrastés, les coûts d'exploitation ne peuvent en aucun cas être considérés comme exogènes mais plutôt comme la résultante d'un certain nombre de facteurs économiques.

Les coûts d'exploitation sont tout d'abord affectés par les fluctuations de la vitesse commerciale du réseau. Une dégradation de cette dernière entraîne une augmentation des charges supportées par les opérateurs. Or, cette dégradation est maintenant en constante progression. L'usage croissant du trafic des véhicules privés détériore fortement l'efficacité productive des opérateurs du transport public qui, en 
définitive, crée une externalité positive dont l'internalisation par le secteur public peut justifier le principe de la subvention.

L'estimation des coûts variables moyens a montré l'insuffisance du niveau des recettes moyennes et donc des prix mis en place par les autorités locales et a montré l'impossibilité d'obtenir une couverture des coûts d'exploitation par les recettes exclusivement issues du trafic. Il a souvent été avancé que le transport par véhicules privés est lourdement subventionné, affectant ainsi négativement les parts de marché du transport des individus et la structure tarifaire du transport public. Une approche idéale pour une éventuelle régulation des déplacements par véhicules privés serait peut être d'imposer une tarification au coût marginal sous la forme de péages qui prendraient en considération les coûts sociaux de l'automobile imposés par les congestions du trafic, la pollution de l'air, le bruit, les accidents, la détérioration des routes et l'utilisation associée des véhicules des services municipaux. De telles taxes augmenteraient considérablement le coût d'utilisation des usagers des véhicules privés tandis que les prix du transport public pourraient augmenter. Ainsi, le besoin de subventionner le secteur pourrait être réduit.

En raison de la congestion des réseaux liée à l'accroissement régulier du trafic, les opérateurs utilisent des véhicules supplémentaires afin d'assurer la ponctualité du service. la quantité associée de facteurs de production nécessaire pour assurer le service est donc supérieure à son niveau d'équilibre. Ceci entraîne une hausse conséquente des coûts d'exploitation, principalement lorsque l'exploitant subi des contraintes financières faibles, à savoir dans le cas des réglementations de type coût $d u$ service. L'instauration de régimes contractuels de type prix fixe entraîne chez l'exploitant une incitation à la réduction des coûts qui ne doit pas être négligée.

Les résultats appellent une formalisation plus structurelle de l'incidence des schémas réglementaires. En s'inspirant de l'approche suggérée dans la section 2.2, il s'agira d'estimer une fonction de coût structurelle directement issue des fonctions d'utilité des opérateurs dans chaque cas et des paramètres d'efficacité et d'effort de productivité non observables par l'économètre. Il s'agira également d'expliquer comment le choix des formes contractuelles est influencé par la nature et le pouvoir de décision des opérateurs et la couleur politique de l'autorité en place et de montrer par une approche dynamique comment le processus de renouvellement des concessions, tel qu'il existe actuellement, conduit à une tendance à la hausse des coûts d'exploitation.

Face à la dérive actuelle des coûts d'exploitation, il serait sans doute nécessaire de réviser l'organisation actuelle des transports urbains en responsabilisant financièrement un peu plus les opérateurs. Une déréglementation accompagnée d'une privatisation générale du service, telle qu'elle fût pratiquée en Grande-Bretagne en $1985,{ }^{34}$ conduirait sans doute à une baisse conséquente des coûts d'exploitation. Entre 1980 et 1994, la déréglementation Britannique a conduit à une réduction des coûts de $30 \%$ malgré un accroissement de l'offre de 12.9\%. Malheureusement, une déréglementation est certainement contraire au principe de service public car, pour ne retenir que l'exemple anglais, la baisse des coûts s'est accompagnée, en plus d'une diminution du niveau de l'emploi de la branche transport urbain (-26\%), d'une détérioration importante de la qualité du service. Les opérateurs en place se sont contentés d'exploiter les lignes les plus rentables et les prix ont subi une augmentation de $31.8 \%$. Ainsi, au cours de la période, la demande a diminué de $26.2 \%$.

A la vue des résultats présentés dans cette étude, il apparaît nécessaire de conserver le statut monopolistique des opérateurs. Mais il semble avant tout 
fondamental de redéfinir les objectifs financiers des firmes, ceci passe par une capacité d'expertise crédible, c'est à dire une bonne connaissance de la technologie en place, des capacités productives et de l'efficacité des opérateurs. A partir du moment où de tels objectifs auront été atteint, la mise en place de structures contractuelles de type prix fixe s'avérera parfaitement pertinente et efficace.

\section{REFERENCES}

Berechman J. (1983). "Analysis of Costs, Economies of Scale and Factor Demand in Bus Transit", Journal of Transport Economics and Policy, vol. 17, pp. 1-16.

Berechman J. (1993). Public Transit Economics and Deregulation Policy, Editions North Holland.

Berndt E.R. (1990). The Practice of Econometrics-Classic and Contempory, Editions Addison-Wesley.

Berndt E.R. et Christensen L.R. (1979). "Parametric Productivity Measurement and Choice Among Flexible Functional Forms", Journal of Political Economy, vol. 87, pp. 1220-1245.

Bessone M. (1995). "Gestion des services publics de transport en commun urbain en 1994", Document de travail, GART.

Blackorby C. et Russel R.R. (1989). "Will the Real Elasticity of Substitution Please Stand Up? A Comparaison of the Allen-Uzawa and Morishima Elasticities", The American Economic Review, 1989, vol. 79, pp. 882-888.

Caillaud B. et Quinet E. (1993). "Analyse du caractère incitatif des contrats de transport urbain", Document de travail, CERAS.

Christensen L.R., Jorgenson D.W. et Lau L.J. (1973). "Transcendental Logarithmic Production Frontiers", Review of Economics and Statistics, vol. 55, pp. 28-45.

Croissant Y. (1996). "Les performances des firmes françaises de transport urbain", Revue d'Economie Politique, vol. 3, pp. 465-485.

Croissant Y. (1997). "Estimation d'une fonction de demande de transport urbain sur données de panel", Document de travail.

Dalen D. et Gomez-Lobo A. (1995). "Incentives Contrats: An Empirical Investigation of the Norvegien Transport Bus Industry", Document de travail, IFS.

Diewert W.E. et Wales T.J. (1987). "Flexible Functional Forms and Global Curvature Conditions", Econometrica, vol. 55, pp. 43-68.

Domenach O. (1987). "Métamorphose des rapports contractuels dans les transports publics urbains", Transports, vol 326.

Fawkner J. (1995). "Le bilan de la déréglementation des transports en GrandeBretagne", Document de travail.

Gagnepain P. et Ivaldi M. (1998). "Current Versus Optimal Regulatory Schemes: Evidence from the French Urban Transport Networks", Document de travail, GREMAQ.

Greene W.H. (1983). "Simultaneous Estimation of Factor Substitution, Economies of Scale, Productivity, and Non-Neutral Technical Change", Developments in Econometric Analyses of Productivity.

Hanoch G. (1975). "The Elasticity of Scale and the Shape of Average Costs", American Economic Review, vol.65, pp. 492-497.

Kerstens K. (1996). "Technical Efficiency Measurement and Explanation of French Urban Transport Companies", Transportation Research A, vol. 30, pp. 431-452. 
Laffont J.J. (1994). "The new Economics of Regulation Ten Years After", Econometrica, vol.62, pp. 507-537.

Laffont J.J. et Tirole J. (1993). A Theory of Incentives in Procurement and Regulation, Editions MIT Press.

Magnus J.R. (1982). "Multivariate Error Components Analysis of Linear and Nonlinear Regression Models by Maximum Likelihood", Journal of Econometrics, vol.19, pp. 239-285.

Mathios A.D. et Rogers R.P. (1989). "The Impact of Alternative Forms of State Regulation of AT\&T on Direct-Dial, Long-Distance Telephone Rates", RAND Journal of Economics, vol. 20, pp. 437-453.

Ohta M. A. (1996). "Note on the Duality Between Production and Cost Functions: Returns to Scale and the Rate of Technological Progress", Economic Studies Quarterly, vol. 25, pp. 63-65.

Wunsch P. (1996). "Cost and Productivity of Major Urban Transit Systems in Europe", Journal of Transport Economics and Research, may, pp. 171-186. 


\section{NOTES}

${ }^{1}$ Je remercie Marc Ivaldi, Jérôme Foncel, Andres Gomez-Lobo et deux rapporteurs anonymes pour leurs commentaires.

${ }^{2} 84 \%$ de l'ensemble.

${ }^{3}$ Dont le financement est garanti par les utilisateurs du réseau.

${ }^{4}$ A l'échelle des transports publics urbains, l'A.O. est la commune, un ensemble de communes ou un regroupement sous forme de districts, de syndicats mixtes ou autres. L'Etat encadre la politique des Autorités Organisatrices qui organisent le réseau de transport : A de rares exceptions qui dépendent du pouvoir de marchandage de l'exploitant et donc de l'importance du groupe auquel il appartient, l'A.O. détermine le prix et le volume de l'offre de transport mais aussi sa capacité et la fréquence de passage. Elle est également responsable de la réalisation et de la gestion des infrastructures.

${ }^{5}$ Les exploitants sont des entreprises qui elles-mêmes peuvent être des sociétés privées, des sociétés d'économie mixte ou des régies. Toutes ces entreprises appartiennent à des groupes plus ou moins importants : $65 \%$ d'entre elles font partie de deux groupes privés (VIA-GTI et CGEA-CGFTE) et d'un groupe semi-public (TRANSDEV), le reste appartenant à des groupes plus modestes (VERNEY-CARIANE et AGIR). Selon M. Bessone (1995), il existe une corrélation non négligeable entre le type de rémunération du contrat que nous décrivons dans cette section et le groupe de transport: En 1995, VIA-GTI regroupait des entreprises liées essentiellement par des contrats de type prix forfaitaire (41\%) ou gérance (34\%) tandis que CGEA avait recours à des contrats de type gérance (60\%) ou des contrats à compensation forfaitaire (32\%). Très souvent, un groupe choisit d'exploiter un réseau pour pénétrer un nouveau marché ou un territoire. Ces motivations, bien souvent stratégiques, peuvent ne pas cadrer avec les objectifs de maximisation du bien-être social de l'A.O.

${ }^{6}$ Il s'agit de l'espace géographique desservi par le réseau.

${ }^{7}$ En moyenne, les recettes sont inférieures aux coûts de moitié.

${ }^{8}$ Elle est financée par trois sources distinctes : Le versement transport payé par les employeurs de plus de neuf salariés, les subventions versées par l'Etat et celles versées par les collectivités locales.

${ }^{9}$ D'autres contrats plus marginaux sont utilisés comme les régies (6\% en 1995), les contrats de type garantie de recettes (3\% en 1995) ou les concessions de travaux et d'exploitation. Nous ne les considérerons pas ici car ils sont fortement minoritaires.

${ }^{10}$ La durée moyenne d'une convention est de cinq ans. Toutefois, le prélèvement des recettes commerciales et le remboursement (partiel/total) des coûts peut être effectué en général à la fin de chaque année, semestre ou mois.

${ }^{11}$ Dans ce cas là, il les conserve.

${ }^{12}$ Et non pas la totalité comme dans le cas des contrats de type gestion à compensation forfaitaire.

${ }^{13}$ Les travaux empiriques ne se sont pas systématiquement concentrés sur les coûts. Dans le cas du téléphone aux Etats-Unis, par exemple, Mathios et Rogers (1989) proposent une approche qui compare le niveau des prix appliqués dans les Etats où les schémas de type taux de rendement sont utilisés aux Etats où une régulation par le prix plafond est employée. La variable expliquée est le ou les prix pratiqués par les différents Etats. Les variables explicatives incluent notamment une variable muette qui prend la valeur 1 si l'Etat autorise une flexibilité des prix à travers un régime de prix plafond. 
L'analyse suggère que les prix pratiqués sont généralement plus faibles de 7 à $13 \%$ dans les Etats où une régulation par le prix plafond est autorisée.

${ }^{14}$ Seuls les périmètres urbains de plus de 100000 habitants ont été sélectionnés. Lyon, Paris et Marseille ne font pas partie de la base de données. Ainsi, la présence potentielle d'hétéroscédasticité due aux différences de taille des réseaux est réduite.

${ }^{15} 431$ au total.

${ }^{16}$ Centre d'Etude et de Recherche sur le Transport Urbain.

${ }^{17}$ Groupement des Autorités Responsables de Transport.

${ }^{18} \mathrm{Si}$ une personne travaille dans l'entreprise durant les huit premiers mois de l'année puis se fait remplacer par une autre personne durant les quatre derniers mois par exemple, l'équivalent personnes-année est égal à 1.

${ }^{19}$ Dans l'industrie du transport, le niveau de production est communément mesuré par les quantités véhicules-kilomètres ou places-kilomètres (Berechman, 1993, par exemple).

${ }^{20}$ Pour chaque véhicule et chaque ligne, il suffit de ramener la distance quotidienne au temps de parcours nécessaire. La moyenne de toutes les lignes et de tous les véhicules sur une année donne la vitesse commerciale du réseau.

${ }^{21}$ A Besançon, au cours de la seconde moitié des années 80 , une détérioration de la vitesse commerciale de $1.5 \mathrm{~km} / \mathrm{h}$ a entraîné une hausse des coûts égale à 6 Millions de Francs.

${ }^{22}$ Voir Caillaud et Quinet (1993).

${ }^{23}$ Dalen et Gomez-Lobo (1995) avaient défini trois facteurs de production dans leur étude sur le transport norvégien et donc trois types de charges : Les salaires versés aux conducteurs représentaient $75.1 \%$ des coûts totaux contre $13.7 \%$ pour les salaires du personnel administratif et $11.2 \%$ pour le fuel.

${ }^{24}$ Les $13.5 \%$ restant correspondent à d'autres types minoritaires ou des données manquantes.

${ }^{25}$ Assimilable en quelque sorte à la taille de chaque firme.

${ }^{26}$ Voir Caves et al. (1981) par exemple.

${ }^{27}$ Voir Diewert et Wales (1987) pour les conditions générales de concavité.

${ }^{28}$ C'est à dire l'équation de coût et une équation de part de coûts.

${ }^{29}$ Pour plus de détails, voir Blackorby et Russell (1989).

${ }^{30}$ Dans le même contexte de l'industrie du transport urbain Français et à partir d'une base de donnée identique, Gagnepain et Ivaldi (1998) considèrent un système séquentiel coûts d'exploitation-capacité-demande de transport qui se présente comme

$$
\left\{\begin{array}{c}
C V=g(y) \\
y=\phi(d) \\
d=d(p)
\end{array}\right.
$$

où $g(),. d($.$) et \phi($.$) sont respectivement des fonctions de coût variable, de demande et$ d'ajustement de l'offre à la demande. Cette approche distingue ainsi la demande de transport $d$ des voyageurs de l'offre de transport $y$ proposée par l'opérateur. On y suppose que l'industrie est caractérisée par un niveau de demande $d$, exprimé par un nombre annuel de voyageurs, qui dépend du prix du service $p$ instauré par l'autorité organisatrice. L'opérateur ajuste sa capacité y à la demande et propose un service qui conduit aux coûts d'exploitation $C V$. De là, les coûts d'exploitation peuvent être 
directement exprimés en fonction de la demande de transport, $C V=g[\phi(d)]$, et le coût marginal d'un voyageur supplémentaire est

$$
\frac{\partial C V}{\partial d}=\frac{\partial g}{\partial y} \phi^{\prime}(d)
$$

On utilise ici l'estimation de $\phi$ obtenue dans Gagnepain et Ivaldi (1998) pour dériver les résultats présentés dans la table 5 et les figures 1 et 2 .

${ }^{31}$ Le prix est alors calculé à partir de la distance parcourue.

${ }^{32}$ Pour cela, on divise simplement les coûts d'exploitation par le nombre de voyageurs transportés.

${ }^{33}$ Les schémas réglementaires peuvent également interférer sur la qualité du service proposé par les opérateurs. Comme il a été spécifié dans la section 2, la qualité du service dépend grandement de la vitesse commerciale du réseau puisque c'est elle qui définit la durée d'un trajet entre deux points. Le temps d'attente au point de départ constitue également un critère important de la qualité du service. Or, le temps d'attente dépend de l'offre de l'exploitant. Plus le nombre de kilomètres et/ou véhicules offerts est important plus le délai d'attente est faible. Comme un plus grand nombre de véhicules-kilomètres offerts à demande donnée est coûteux, il est vraisemblable que les régimes contractuels qui assurent un équilibre budgétaire final certain inciteront les opérateurs à fournir une qualité de service supérieure. Inversement, dans le cas des régimes contractuels plus risqués de type prix-fixe, on peut s'attendre à une réduction des coûts en raison d'un niveau de qualité plus faible à demande donnée, donc, là encore, à un effet négatif de la variable $P F$ sur les coûts.

${ }^{34}$ Voir Berechman (1993) et Fawkner (1995). 
Figure 1 : Coûts marginaux estimés, coûts variables moyens estimés et recettes moyennes calculées, 1985

1985

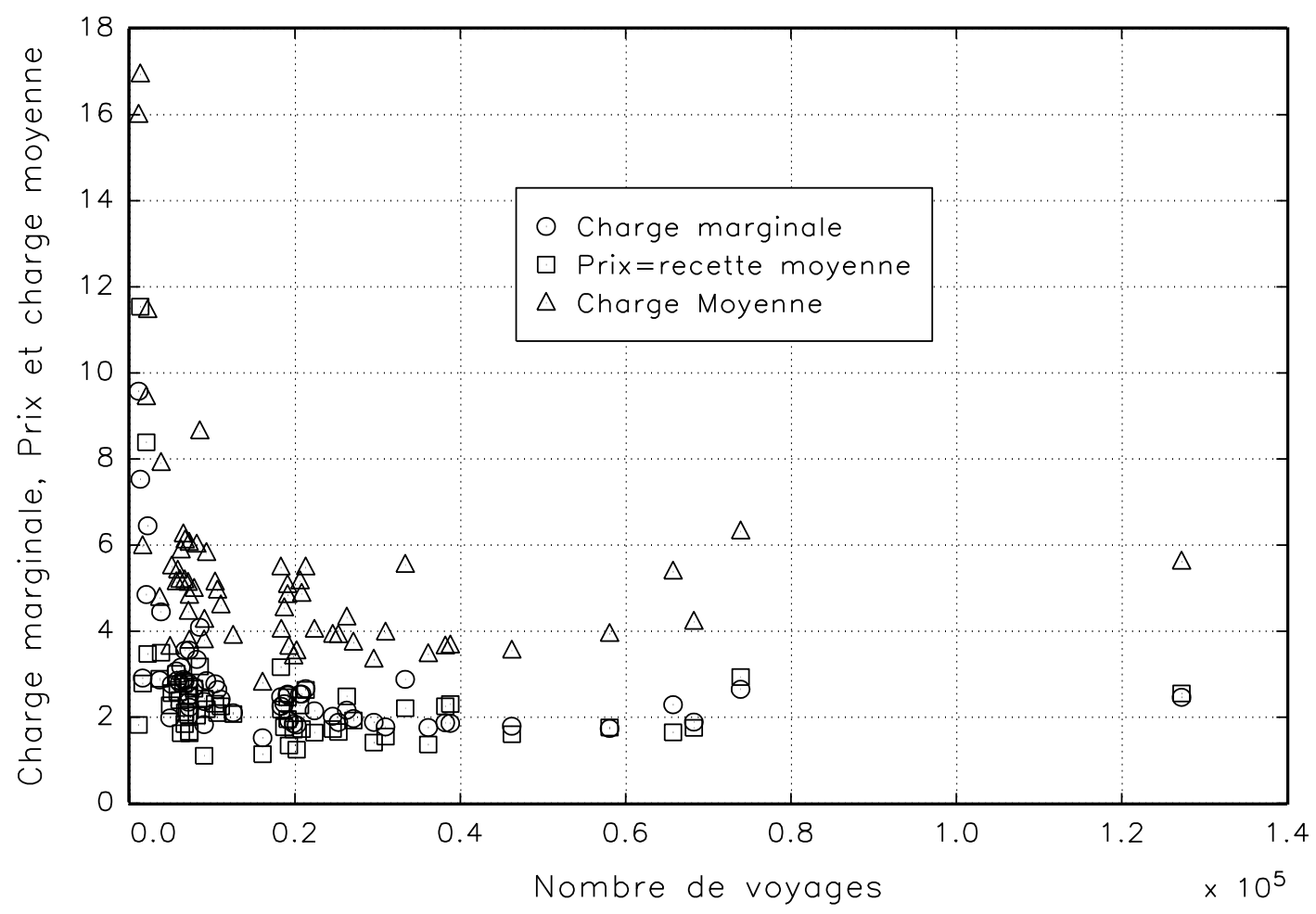

Figure 2 : Coûts marginaux estimés, coûts variables moyens estimés et recettes moyennes calculées, 1985-1993

$1985-1993$

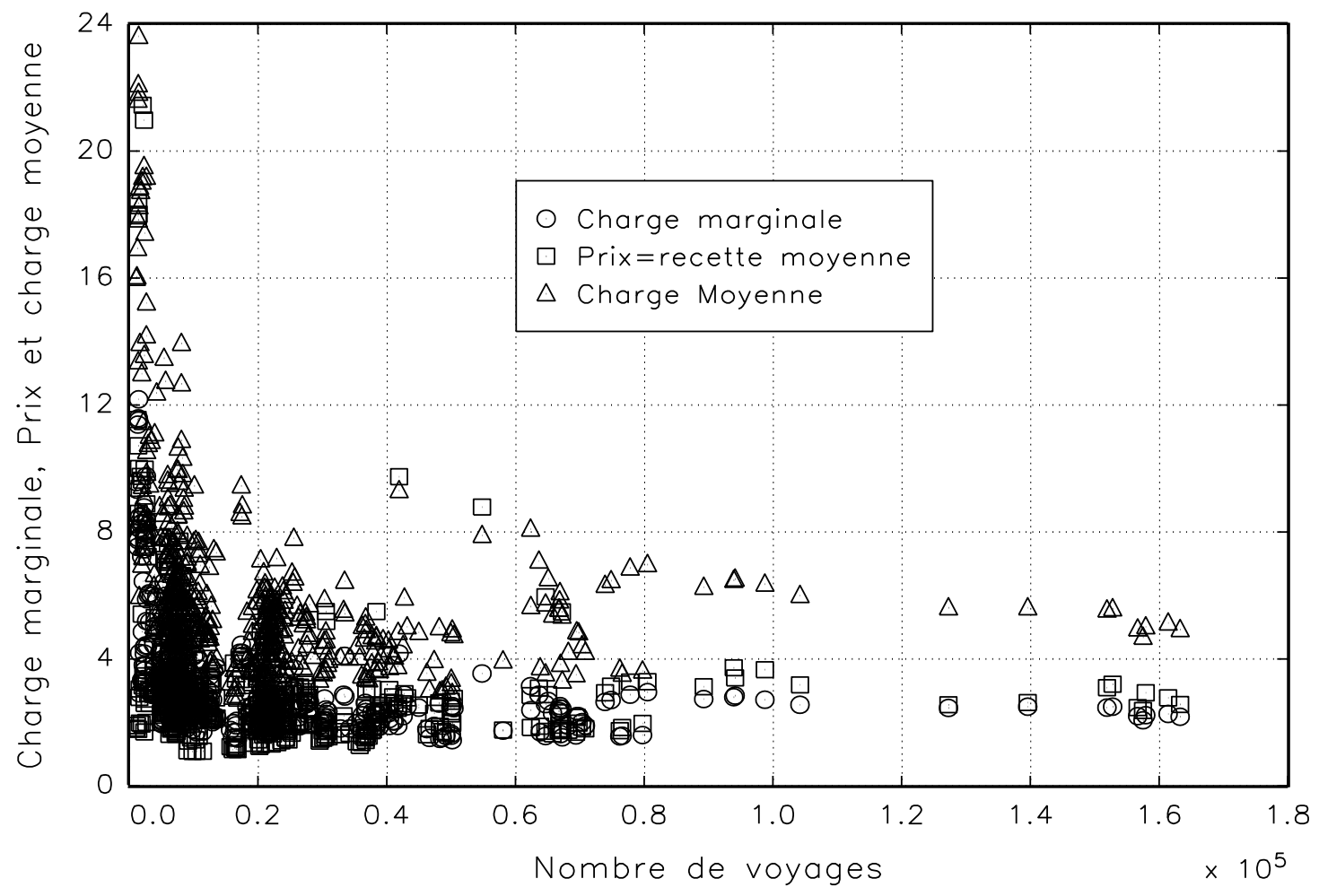


Tableau 1

\begin{tabular}{lrrr}
\hline Variables & Moyenne & Ecart-type & Quantité \\
\hline \hline Prix du travail & 174,94 & 28,364 & \\
Prix de l'énergie. & 2,857 & 387 & \\
Kilomètres offerts & $5,388,601$ & $5,452,982$ & \\
Capacité totale & 144,14 & 138,763 & 236 \\
Vitesse commerciale & 16.676 & 2,654 & 116 \\
Contrats de gérance & & & \\
Contrats type prix forf. & & & \\
Contrats type comp.forf. & 229,631 & & \\
Population totale & $125,660,000$ & $142,182,000$ & \\
Coûts totaux & $73,448,000$ & & \\
Recettes commerciales & $52,670,000$ & & \\
Subventions & 0.898 & & \\
Part salaires & 0.102 & & \\
Part énergie & & & \\
\hline Note & & & \\
\hline
\end{tabular}

Note: Les recettes commerciales sont les recettes issues de la vente du service transport exclusivement. Les subventions sont les sommes versées à l'A.O. qui équilibrent le compte transport de l'ensemble du réseau à l'issue de la période.

Prix et coûts indiqués en Francs

Tableau 2

\begin{tabular}{lcc}
\hline Variable & Estimation & Ecart-Type \\
\hline \hline & & \\
$C s t e$ & 5.8342 & 0.8608 \\
$\ln (p L)$ & 0.5349 & 0.0193 \\
$\ln (y)$ & 1.1514 & 0.2427 \\
$\ln (K)$ & -2.0199 & 0.4377 \\
$\ln I N F$ & 0.0923 & 0.2103 \\
$V I T$ & -0.1316 & 0.0295 \\
$t$ & -0.0062 & 0.0010 \\
$P F$ & -0.0205 & 0.0078 \\
& & \\
& & \\
$\ln (p L) \ln (p L)$ & & \\
$\ln (y) \ln (y)$ & 0.0786 & 0.0023 \\
$\ln (p L) \ln (y)$ & 0.0486 & 0.0210 \\
$\ln (I N F) \ln (I N F)$ & 0.0045 & 0.0023 \\
$\ln (y) \ln (I N F)$ & -0.0815 & 0.0315 \\
$\ln (K) \ln (K)$ & -0.0737 & 0.0284 \\
$\ln (p L) \ln (K)$ & 0.2099 & 0.0629 \\
$\ln (p L) \ln (I N F)$ & 0.0010 & 0.0030 \\
$\ln (K) \ln (y)$ & -0.0019 & 0.0025 \\
$\ln (K) \ln (I N F)$ & -0.0883 & 0.0333 \\
$\quad$ Nombre d'observations & 0.1952 & 0.0501 \\
\hline
\end{tabular}


Tableau 3

\begin{tabular}{|l|l|l|}
\hline & Travail & Energie \\
\hline Parts observées & 0.898 & 0.102 \\
\hline Parts estimées & 0.897 & 0.103 \\
\hline
\end{tabular}

Tableau 4

\begin{tabular}{|l|l|l|}
\hline & Travail & Energie \\
\hline Travail & -0.0153 & 0.1491 \\
\hline Energie & 0.1491 & -0.1338 \\
\hline
\end{tabular}

Tableau 5

\begin{tabular}{|l|l|l|}
\hline Prix-Recette moyenne & Coût marginal & Coût moyen \\
\hline 3.01 & 2.80 & 6.08 \\
\hline
\end{tabular}

